\title{
Structural properties of amorphous hydrogenated silicon using ab initio molecular dynamics simulations
}

\author{
GIRISH R GUPTE, R PRASAD, VIJAY KUMAR* and \\ G L CHIAROTTI ${ }^{\dagger}$ \\ Department of Physics, Indian Institute of Technology, Kanpur 208 016, India \\ * Indira Gandhi Centre for Atomic Research, Kalpakkam 603 102, India \\ 'International School of Advanced Studies (SISSA), Trieste, Italy
}

\begin{abstract}
We have studied structural properties of amorphous hydrogenated silicon using $a b$ initio molecular dynamics simulations. A sample was generated by simulated annealing using periodic boundary conditions with a supercell containing 64 silicon and 8 hydrogen atoms. The radial pair distribution functions for $\mathrm{Si}-\mathrm{Si}, \mathrm{Si}-\mathrm{H}$ and $\mathrm{H}-\mathrm{H}$ have been studied at $300 \mathrm{~K}$ and are found to be in good agreement with experimental data. Our results show that hydrogen saturates the dangling bonds and reduces bond strain. We also report existence of $\mathrm{Si}-\mathrm{H}-\mathrm{Si}$ bridge sites which are likely to play an important role in understanding the light induced metastability in this material.
\end{abstract}

Keywords. Amorphous hydrogenated silicon; radial distribution function; $a b$ initio molecular dynamics; bond angle distribution.

\section{Introduction}

Amorphous hydrogenated silicon ( $a-\mathrm{Si}: \mathrm{H})$ is an important material for the development of photovoltaic devices. However, it suffers from light induced metastable changes (the Staebler-Wronski effect) (Staebler and Wronski 1980) which are believed to lead to an increase in the number of defects and the associated increase in the number of localized states in the gap of the material which shortens the recombination time for electron-hole pairs (Street 1991). In $a$-Si:H hydrogen plays an important role in the passivation of the dangling bonds and reduction of the density of localized states. The light induced metastability seems to be closely linked with the anomalous diffusion of hydrogen and its proper understanding is a topic of much current interest. While several efforts have been devoted to understand this phenomenon, the situation is still far from clear (Yiping et al 1995). Here we present results of an ab initio molecular dynamics study of the structure of $a$-Si:H which shows the presence of weakly bonded bond centred $(\mathrm{BC}) \mathrm{H}$ which could play an important role in the anomalous diffusion of $\mathrm{H}$.

\section{Method}

We have used the Car-Parrinello (1985) method to study the structural properties of $a$-Si:H. The electron-ion interaction for Si was represented by the norm-conserving pseudopotentials of Bachelet et al (1982) with $s$ and $p$ non-locality in the Kleinmann and Bylander (1982) form. However, for hydrogen the actual $1 / r$ potential was used. The wave functions were expanded in a plane wave basis with kinetic energy up

*Author for correspondence 
to $12 \mathrm{Ry}$ and $\mathbf{k}=0$ point was used for the Brillouin zone sampling. The equations of motions were integrated using the Verlet algorithm with a time step of $1.209 \times 10^{-16} \mathrm{sec}$ and electronic mass of 200 . Periodic boundary conditions were imposed with a simple cubic supercell of side $10 \cdot 862 \AA$ which was kept constant throughout the simulation. The supercell contained $64 \mathrm{Si}$ and $8 \mathrm{H}$ atoms. The $a-\mathrm{Si}: \mathrm{H}$ sample was prepared using simulated annealing starting with a random displacement of $\mathrm{Si}$ atoms on a diamond structure and $\mathrm{H}$ atoms on the $\mathrm{BC}$ sites. The sample was heated to $2200 \mathrm{~K}$ and equilibrated for roughly 5000 time steps. It was then cooled to $1000 \mathrm{~K}$ in steps of $200 \mathrm{~K}$ with roughly 2000 time steps at each temperature. Then the sample was cooled to $300 \mathrm{~K}$ in steps of $100 \mathrm{~K}$ with 1000 time steps for each temperature. At $300 \mathrm{~K}$ the sample was equilibrated for roughly 5000 time steps. After this the time averaged structural functions were calculated over a time span of about 5000 steps.

\section{Results and discussion}

\subsection{Radial pair distribution functions}

Figure 1 shows the time averaged radial distribution functions for $\mathrm{Si}-\mathrm{Si}, \mathrm{Si}-\mathrm{H}$ and H-H. The Si-Si radial distribution function shows a sharp peak centred at $2 \cdot 37 \AA$ corresponding to the nearest neighbours and a broader peak at $3 \cdot 72 \AA$ related to next nearest neighbours in close agreement with the experimental values of 2.35 and $3.7 \AA$ (Menelle 1987). The $\mathrm{Si}-\mathrm{H}$ pair distribution function shows a sharp peak centred at $1.59 \AA$ corresponding to nearest neighbours and broader peaks related to next and next

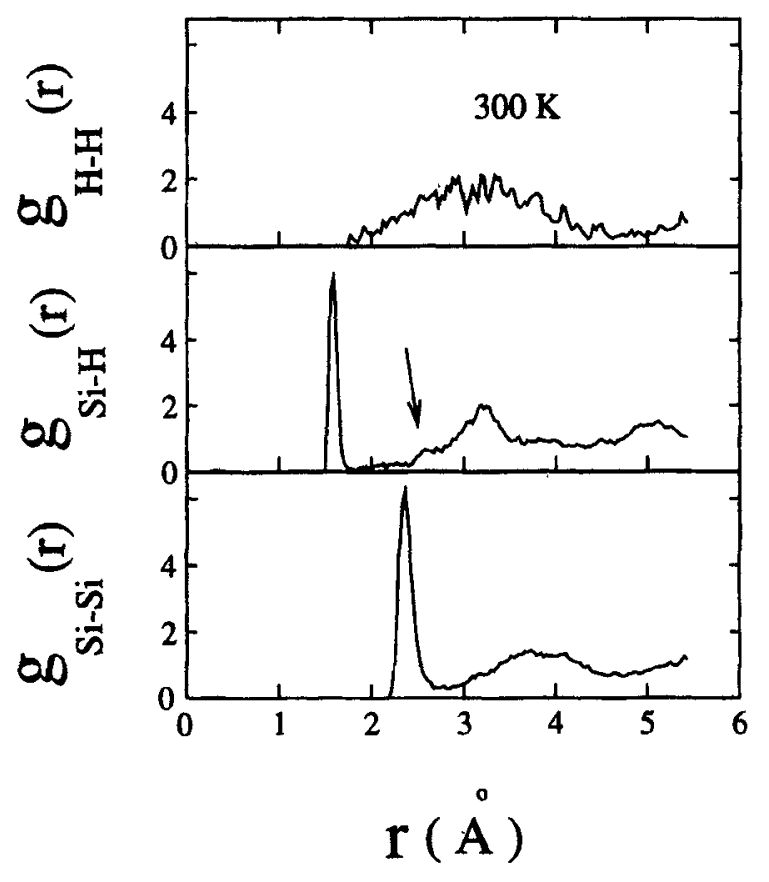

Figure 1. Radial pair distribution function for hydrogenated $a$-Si. 


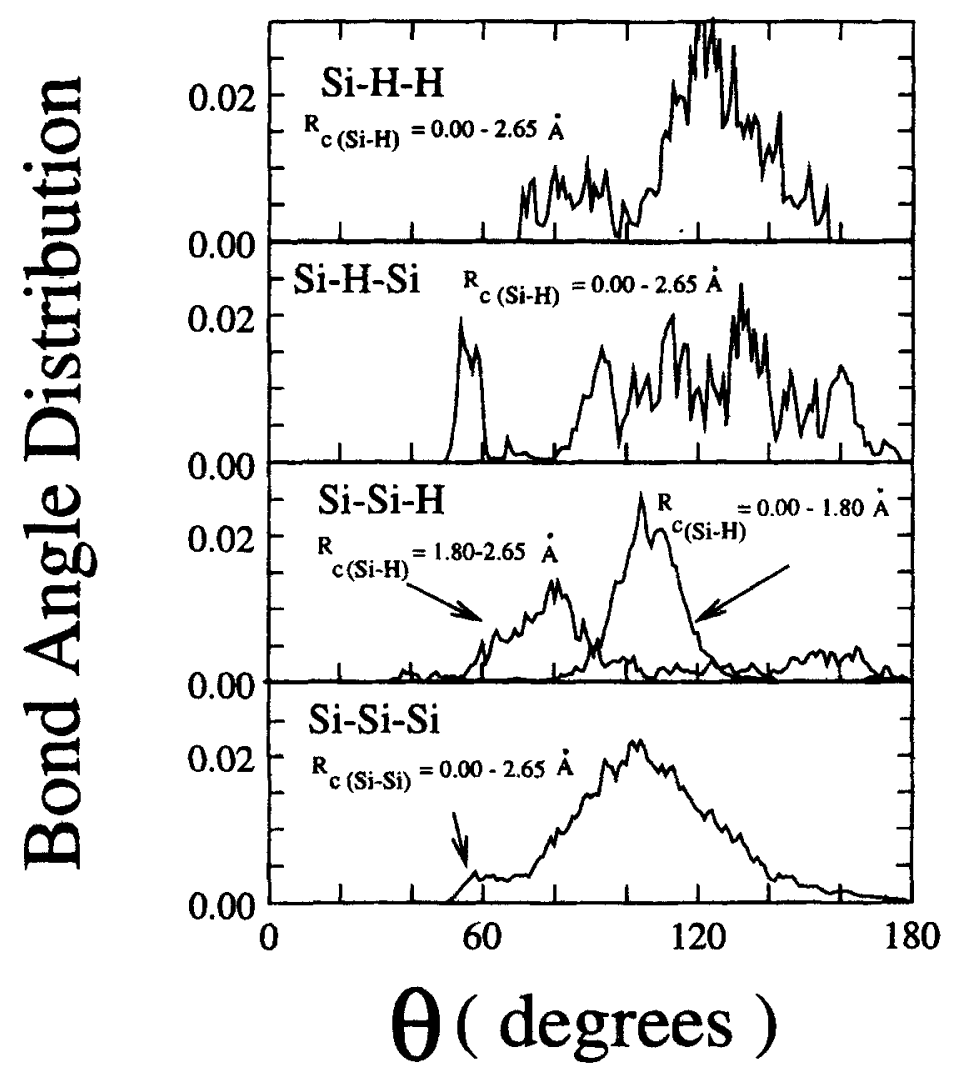

Figure 2. Bond angle distribution for hydrogenated $a-\mathrm{Si}$.

to next nearest neighbours centred at $3 \cdot 18 \AA$ and $5 \cdot 25 \AA$ respectively. This also agrees well with the experimental values (Menelle 1987) of 1.59, 3.1 and 4.95 $\AA$ and the results of a previous calculation (Buda et al 1990, 1991). However, we obtained a shoulder at around $2 \cdot 50 \AA$. This feature corresponds to a floating $\mathrm{Si}-\mathrm{H}$ bond and is seen in the experimental data (Menelle 1987) but not in the earlier calculation (Buda et al 1990, 1991). The $\mathbf{H}-\mathbf{H}$ distribution function is broad lying between 2 to $4 \AA$ and therefore in our sample we do not have molecular form of hydrogen normally seen in device grade $a$-Si:H.

\subsection{Bond angle distribution}

Figure 2 shows the bond angle distributions. The cut-off radius for $\mathrm{Si}-\mathrm{Si}$ was taken to be $2.65 \AA$ i.e. just after the first peak in $\mathrm{Si}-\mathrm{Si}$ radial distribution function. The cut-off radius for $\mathrm{Si}-\mathrm{H}$ was also fixed to $2.65 \AA$ as we were interested in the weak $\mathrm{Si}-\mathrm{H}$ bonds. For $\mathrm{Si}-\mathrm{H}$ bonding we used a minimum as well as a maximum cut-off. Only those $\mathrm{Si}-\mathrm{H}$ pairs which were separated by a distance more than the minimum value and less than the maximum value are counted as bonds. The bond angle distribution for $\mathrm{Si}-\mathrm{Si}-\mathrm{Si}$ shows a broad peak centred around $108^{\circ}$, showing a 4 -fold coordination. But also 
present is a shoulder at around $60^{\circ}$ which indicates presence of $\mathrm{Si}$ atoms which are not 4-fold coordinated. Indeed the average coordination number $(\mathrm{CN})$ of $\mathrm{Si}$ atoms is $\mathbf{4 . 2 8}$ when cut-off radius for $\mathrm{Si}-\mathrm{H}$ bond is taken to be $2.65 \AA$. When this is reduced to $1.8 \AA$, the $\mathrm{CN}$ reduces to $4 \cdot 18$, but this also is significantly more than 4 . Our analysis shows that in addition to 4 -fold coordinated sites $(71.7 \%)$, there are a large number of $\mathrm{Si}$ atoms which are 3 -fold ( $3 \%$ ), 5 -fold $(20 \cdot 2 \%)$ and 6 -fold (5.1\%) coordinated. If the $\mathrm{Si}-\mathrm{H}$ bond length is restricted to $1.8 \AA$, then the percentage of 4-fold coordinated sites increases to 79 predominantly at the expense of the 5-fold sites. Coordination number for hydrogen is very sensitive to the variation in cut-off radius for $\mathrm{Si}-\mathrm{H}$ bond. The average CN of hydrogen is $2 \cdot 10$ when $R_{\mathrm{c}}(\mathrm{Si}-\mathrm{H})$ is $2 \cdot 65 \AA$, but it falls to $1.31 \AA$ when $R_{\mathrm{c}}(\mathrm{Si}-\mathrm{H})$ is reduced to $1.8 \AA$. But even this is significantly more than 1 indicating that $\mathrm{H}$ can form bonds with two or more atoms. We find that more than half of $\mathrm{H}$ atoms have two nearest neighbours within a radius of $2.65 \AA$. When $R_{\mathrm{c}}(\mathrm{Si}-\mathrm{H})$ was kept at $1.8 \AA$, this number reduces to $30 \%$ but it is still significant. This also means that there are significant number of $\mathrm{H}$ atoms which are bound to one $\mathrm{Si}$ atom and are within $2.65 \AA$ from another $\mathrm{H}$ atom. There are a significant number of $\mathrm{Si}$ atoms with $3 \mathrm{Si}$ and one strongly bound $H$ with bond length less than $1.8 \AA$. Also from figure 2 the distribution of bond angles between $\mathrm{Si}-\mathrm{Si}$ bond and $\mathrm{Si}-\mathrm{H}$ bond with bond length less than $1.80 \AA$ is centred around $108^{\circ}$. This peak is relatively sharper than the corresponding peak for the angle between two $\mathrm{Si}-\mathrm{Si}$ bonds. This indicates that hydrogen not only saturates the dangling bonds but also reduces bond strains. This is likely to lead to an increase in the band gap. The weak $\mathrm{Si}-\mathrm{H}$ bonds with bond lengths between $1.80 \AA$ to $2.65 \AA$ show two peaks at $83^{\circ}$ and $160^{\circ}$, while there are almost no bond angles near $108^{\circ}$ showing that these bonds are not 4-fold coordinated. The presence of bond angles near $160^{\circ}$ indicates presence of nearly linear $\mathrm{Si}-\mathrm{Si}-\mathrm{H}$ formations. The bond angle distribution for $\mathrm{Si}-\mathrm{H}-\mathrm{Si}$ has several peaks between $80^{\circ}$ and $180^{\circ}$ and one sharp peak at $60^{\circ}$. The peaks between $80^{\circ}$ and $180^{\circ}$ indicate that hydrogen has local minimum energy sites between two Si atoms not directly bound. Our simulations show that hydrogen spends more time in these energy pockets. It has been shown (Fedders and Drabold 1993) that the binding energy of $\mathrm{H}$ on such bridge sites is about $3 \mathrm{eV}$ less as compared to a dangling bond site. Therefore this $\mathrm{Si}-\mathrm{H}-\mathrm{Si}$ bridge site can play an important role in the light-induced metastability (Street 1991; Prasad and Shenoy, to be published). Also $\mathrm{H}$ can have one $\mathrm{Si}$ and one $\mathrm{H}$ nearest neighbours with angle between the $\mathrm{H}-\mathrm{H}$ bond and $\mathrm{H}-\mathrm{Si}$ bond showing a peak at around $120 \AA$ and a small peak near $80^{\circ}$. These $\mathrm{H}-\mathrm{H}$ bonds are weak with bond lengths more than $1.80 \AA$ as can be seen from figure 1 .

\section{Conclusions}

Hydrogen is observed to play an important role in the saturation of the dangling bonds and in the reduction of bond strain in $a-\mathrm{Si}: \mathrm{H}$. Also a fraction of $\mathrm{H}$ atoms occupies bridge site between two $\mathrm{Si}$ atoms which are not directly bound. Such weakly bonded $\mathrm{H}$ could be significant in understanding the anomalous diffusion of $\mathrm{H}$ and the light induced metastability. Our results of the radial distribution function are in very good agreement with experiments and show an improvement over the previous calculations. The effect of such distribution of $\mathbf{H}$ on the electronic properties of $a-S i: H$ and the dynamical behaviour of $\mathrm{H}$ are currently under study and will be published separately. 


\section{References}

Bachelet G B, Hamann D R and Schluter M 1982 Phys. Rev. B26 4199

Buda F, Chiarotti G L, Car R and Parrinello M 1990 Mater. Res. Soc. Symp. Proc. 163383

Buda F, Chiarotti G L, Car R and Parrinello M 1991 Phys. Rev. B44 5908

Car $R$ and Parrinello M 1985 Phys. Rev. Lett. 552471

Fedders P A and Drabold D A 1993 Phys. Rev. B47 13277

Kleinmann L and Bylander D M 1982 Phys. Rev. Lett. 481425

Menelle A 1987 Ph. D. Thesis, Paris

Staebler D L and Wronski C R 1980 J. Appl. Phys. 513262

Street R A 1991 Physica B170 69

Yiping Z, Dianlin Z, Guanglin K, Guangqin P and Xianbo L 1995 Phys. Rev. Lett. 74558 